\title{
Variables que inciden en el desarrollo de las vocalizaciones tempranas: su importancia para la evaluación
}

\author{
Ana Pérez 1, Manuela Martínez-Lorca 2 y Carmen Zabala 2 \\ 1 Centro de atención a personas con discapacidad, AMIBIL, España \\ 2 Universidad de Castilla la Mancha, España
}

\begin{abstract}
Resumen
El objetivo del presente trabajo es conocer las producciones vocales tempranas anteriores a la primera palabra, así como estudiar la influencia de algunos factores extralingüísticos en ellas (edad, sexo, cuidador principal, orden de nacimiento y nivel educativo materno). Para ello, se ha realizado un estudio descriptivo transversal en el que han participado 39 niños/as de ambos sexos en edades comprendidas entre los 8 y 15 meses de edad. Los niños/as acudían a diferentes centros de Educación Infantil públicos y privados de la provincia de Toledo. Como medida se ha empleado el apartado "Vocalizaciones" de la versión española del Inventario de Desarrollo Comunicativo MacArthur-Bates (López-Ornat, Gallego, Gallo, Karousou, Mariscal y Martínez, 2005). Los resultados reflejan que la actividad vocal en los bebés entre 8 y 15 meses de edad aparece gradualmente de forma muy temprana constatándose la influencia de algunas variables extralingǘsticas. Los aspectos prosódicos del lenguaje se relacionan con la variable sexo y el nivel formativo de la madre. La variable orden de nacimiento también muestra una relación con el desarrollo de las vocalizaciones, concretamente en los bebés nacidos en primer lugar. En cuanto a la observación del desempeño comunicativo y lingüístico de los bebés por parte del cuidador principal, se encuentra un sesgo observacional apareciendo una mayor sobreestimación de las vocalizaciones por parte de los cuidadores distintos a la madre.
\end{abstract}

Palabras clave: Factores extralingüísticos; Inventario de Desarrollo Comunicativo MacArthur-Bates; Vocalizaciones.

\section{Variables that affect the development of early vocalizations: their relevance to the assessment.}

\begin{abstract}
The aim of this study was to determine the early vocal productions before the first word, and to study the influence of extra-linguistic factors (age, gender, primary caregiver, birth order, maternal education level). To this end, a cross-sectional descriptive study has been performed which involved 39 children of both sexes aged between 8 and 15 months of age. The children went to different schools of public and private Childhood Education in the province of Toledo. As a result we have used the "vocalizations" part of Inventory MacArthur-Bates Communicative Development (López-Ornat, Gallego, Gallo, Karousou, Mariscal and Martinez, 2005). The data show that the speech activity in infants between 8 and 15 months of age appear gradually very early confirming the influence of some extralinguistic variables. Prosodic aspects of language are related to the variable sex and educational level of the mother. Birth as an order variable also shows a connection with the development of vocalizations, particular in babies born in first place. As for the observation of communicative and linguistic performance of babies by the primary caregiver, there is an observational bias appearing greater overestimation of vocalizations by caregivers different from the mother.

Keywords: Extra-linguistic factors; MacArthur Communicative Development Inventory; Vocalizations.
\end{abstract}


Correspondencia con los autores: manuela.martinez@uclm.es Recibido 12 Mayo. Primera revisión 24 Julio. Aceptado 17 Noviembre.

Agradecimientos: Los resultados de este trabajo han sido posibles gracias a la colaboración de padres y madres cuyos bebés han estado escolarizados en diferentes Centros de Atención a la Infancia (CAI) de la provincia de Toledo (El Alfar de Talavera de la Reina y CAI Castilla-La Mancha de Toledo) y Guarderías como "El bosque de las Hadas" (Toledo) y "Arco Iris" (Talavera de le Reina). A todos ellos nuestro más sincero agradecimiento por su desinteresada colaboración. Por último, agradecemos al editor y a los revisores todas sus valiosas críticas, sugerencias y comentarios para mejorar el presente trabajo.

\section{Introducción}

La adquisición y desarrollo por parte de los niños/as del sistema de sonidos de su lengua nativa evoluciona enormemente en los primeros años de vida. Desde los llantos o los gemidos iniciales, pasan a ser capaces de controlar una gama impresionante de sonidos en pocos meses, para posteriormente comprender y expresar intenciones y significados que forman palabras y oraciones (Mariscal \& Gallo, 2006; Owens, 2003; Serra, Serrat, Solé, Bel, \& Aparici, 2013). A lo largo de este proceso, un buen desarrollo del lenguaje en el niño/a facilita su desarrollo cognitivo y socioemocional, repercutiendo enormemente, así mismo, en su evolución social e intelectual y en su adaptación y éxito escolar (Ambrose, Unflat, Walker, Harrison, Oleson, \& Moeller, 2014; Guiberson, Rodríguez, \& Dale, 2011; Mariscal et al., 2007). De acuerdo con lo anterior, un buen desarrollo lingüístico en los niños/as es un indicador de éxito futuro.

Un elemento importante que parece favorecer el desarrollo lingüístico son las vocalizaciones tempranas. Se trata de emisiones vocales muy abundantes que un bebé produce antes de la producción del lenguaje convencional. Estas vocalizaciones, carecen frecuentemente de formas fonológicas adultas (por ejemplo, de contenido segmental), lo que las hace difícil de explicar, de transcribir y de codificar. Pese a ello, se considera que tienen un alto valor comunicativo concediéndose una especial relevancia a la relación entre este desarrollo vocal y el desarrollo simbólico (López-Ornat \& Karousou, 2005; Mariscal \& Gallo, 2006).

En la actualidad, numerosas investigaciones consideran las vocalizaciones tempranas de los bebés como índices tempranos del desarrollo comunicativo y lingüístico posterior. Ello se debe a su posible valor predictivo de normalidad en los avances evolutivos que se dan en las distintas edades en el domino de las diferentes dimensiones del lenguaje (Bruce, Kornfalt, Radeborg, Hansson, \& Nettelbladt, 2003; D’Odorico, Majorano, Fasolo, Salerni, \& Suttura, 2011; Karousou, 2003; Karousou \& López-Ornat, 2013; López-Ornat \& Karousou, 2005; Oller, 2000; Ramsdell, Oller, Buder, Ethington, 
\& Chorna, 2012; Stoel-Gammon, 2011). Como consecuencia, la evaluación del lenguaje antes de los 30 meses parece constituir una necesidad de la investigación y de la práctica, tanto clínica como educativa, con el fin de detectar lo más tempranamente posible cualquier retraso o trastorno del lenguaje. Ello permitiría identificar niños/as con riesgo e intervenir lo más pronto posible para minimizar los posibles retrasos y maximizar las posibilidades de desarrollo (Bruce et al., 2003; Karousou \& López-Ornat, 2013; Mariscal et al., 2007; Mariscal, Nieva \& López-Ornat, 2010; Ramsdell et al., 2012).

Por otro lado, las pruebas estandarizadas existentes para la evaluación temprana del lenguaje y la comunicación en español son escasas. Además, generalmente no son aplicables a niños/as menores de 3 años y solo evalúan aspectos parciales del lenguaje (Mariscal et al., 2007). Una posible explicación de este hecho es que la evaluación lingüística a estas edades conlleva importantes dificultades metodológicas (Mariscal et al., 2007; Pérez \& García, 2003; Pérez-Pereira \& Resches, 2007).

Una alternativa para la evaluación y detección temprana del lenguaje en los niños/as menores de 3 años es el uso de los inventarios paternos. En principio, podría pensarse que los informes que los padres realizan de las competencias comunicativas y lingüísticas de sus hijos/as pueden presentar cierta subjetividad o diversos tipos de sesgos observacionales (Farkas, 2011; Pérez, \& García, 2003; Pérez-Pereira \& Resches, 2007). Pese a ello, existe bastante consenso en la literatura científica acerca de la alta fiabilidad y validez de los padres y madres como informantes del desarrollo lingüístico típico y atípico de sus hijos/asen la infancia temprana. Se ha demostrado, por ejemplo, una alta correlación entre la observación del desempeño lingüístico de los niños/as evaluado mediante diversas medidas (muestras de lenguaje natural, test de vocabulario, etc.) y la información suministrada por padres o cuidadores acerca de ese desempeño (Bates, Benigni, Bretherton, Camaioni, \& Volterra, 1979; Guiberson et al., 2011; Karousou \& López-Ornat, 2013; Marcham \& Martinez-Sussmann, 2002; Mariscal, Nieva, Gallego, y López-Ornat, 2006; Mariscal et al., 2010; Thal, Jackson-Maldonado, \& Acosta, 2000). Por tanto, los informes paternos representan una buena herramienta sencilla, rápida, económica y muy precisa para conocer las habilidades comunicativas y lingüísticas de los niños y niñas muy pequeños.

Dentro de este contexto, Fenson et al. (1993) fueron pioneros en la creación de un instrumento de cribado (screening) completado por los padres para diferenciar desde muy temprano (entre los 8 y los 30 meses) el desarrollo lingüístico y comunicativo típico del atípico. Este instrumento de medida es el Communicative Development Inventory 
MacArthur-Bates (Inventario de Desarrollo Comunicativo MacArthur-Bates), más conocido como CDI. Este inventario emplea ítems muy concretos siendo la tarea de los padres el reconocimiento de comportamientos lingüísticos y comunicativos de sus hijos/as. Esto supone un coste reducido en términos de tiempo y dinero lo que permite la evaluación del desarrollo comunicativo y lingüístico en muestras muy amplias de sujetos. Por estos motivos, actualmente este instrumento está baremado para más de 25 lenguas, lo que facilita la comparación inter-lenguas (ver http://www.sci.sdsu.edu/cdi/cdiwelcome.htm).

La primera adaptación del inventario de desarrollo comunicativo MacArthurBates para el español fue realizada por Jackson-Maldonado et al. (2003) para el español de México. Para el español de España, la adaptación fue llevada a cabo por el equipo de López-Ornat et al. (2005) modificándola cultural y lingüísticamente y revisándola técnicamente. Otras adaptaciones se han realizado al gallego (Pérez \& García, 2003; Pérez-Pereira \& Resches, 2007), al euskera (García, Arrabitel, Barreña \& Ezeizabarrena, 2008) e incluso también existe una adaptación para los niños/as con síndrome de Down para el español de España (Galeote et al., 2006; Galeote, Soto, Sebastián, Rey \& Checa, 2012). Por tanto, existe bastante evidencia de la alta fiabilidad y validez del CDI para evaluar el desarrollo temprano del lenguaje en diferentes lenguas e incluso en muestras con desarrollo atípico. Por este motivo su uso se ha generalizado en los últimos años para evaluar el desarrollo comunicativo-lingüístico en niños/as pequeños, así como para estudiar las variables extralingüísticas que influyen en el proceso de adquisición del lenguaje.

Por lo que respecta a esas variables, una de las que mayor controversia ha generado es la influencia que ejerce el sexo sobre el desarrollo comunicativo infantil. Algunos autores (Bouchard, Trudeau, Sutton, Boudreault, \& Deneault, 2009; JacksonMaldonado, Marchman, \& Fernald, 2013; Schults, Tulviste, \& Konstabel, 2012) apoyan la superioridad de las niñas frente a los niños en el desarrollo lingüístico posterior, mientras que en otros trabajos (Jackson-Maldonado et al., 2013; López-Ornat et al., 2005; Mariscal et al., 2007; Pérez \& García, 2003; Pérez-Pereira \& Resches, 2007) las niñas no presentan esa superioridad.

En segundo lugar, con respecto a la influencia que ejerce el orden de nacimiento en el desarrollo lingüístico, se observa una cierta ventaja en el desarrollo comunicativolingüístico entre los niños/as nacidos en primer lugar frente a aquellos con hermanos/as mayores (Benigno, Clark, \& Farrar, 2007; Galián, Ato y Carranza, 2010; Hoff, 2006; 
Malmeer \& Assadi, 2013; Pancsofar \& Vernon-Feagans, 2010; Pérez y García, 2003; Pérez-Pereira \& Resches, 2007; Schults et al., 2012). Este hecho se atribuye a que los padres y madres que tienen un solo hijo/a disponen de más tiempo para jugar, interaccionar y poner en marcha múltiples, variados y ricos intercambios comunicativos.

En tercer lugar, una variable que ha de interpretarse con cautela pero que es especialmente relevante por su influencia sobre el desarrollo lingüístico infantil hace referencia al estatus socioeconómico familiar, frecuentemente asociado al nivel formativo y educativo de la madre. Distintos autores (Furey, 2011; Hoff, 2006; Mariscal et al., 2007; Pancsofar \& Vernon-Feagans, 2006; 2010; Pérez \& García, 2003; Schults et al., 2012), han confirmado cómo niveles altos de formación educativa en las madres está relacionado con mayores tasas de comprensión y producción de vocabulario infantil. Ello se debería a que estas madres hablan e interactúan más con sus hijos/as, les proporcionan mayores oportunidades de estimulación lingüística, usan mayor número de gestos mientras hablan con estos y cuando éstos interactúan con sus progenitoras, etc. Por lo que respecta al menor desarrollo lingüístico entre los niños/as de contextos socioeconómicos inferiores, se han dado varias explicaciones: subestimación de las habilidades de producción del vocabulario por parte de las familias con menos recursos socioeconómicos, dificultades de interpretación del instrumento por parte de los padres o bien la existencia, realmente, de un menor desarrollo lingüístico (Furey, 2011; Guiberson \& Rodríguez, 2010; JacksonMaldonado et al., 2013; Pérez-Pereira \& Resches, 2007).

Por último, la madre es generalmente la cuidadora principal y más significativa de los hijos/as siendo con esta con la que pasan la mayor parte del tiempo a lo largo del día. Algunos autores (DePaolis, Vihman, \& Nakai, 2013; Hoff, 2006; Hurtado, Marchman, \& Fernald, 2008; Karousou \& López-Ornat, 2013; Pancsofar \& VernonFeagans, 2006; 2010; Ramsdell et al., 2102) muestran la relación que existe entre la figura de la madre y el desarrollo de las vocalizaciones prelingüísticas. Esta relación se refleja en intensas y frecuentes interacciones entre la madre y el hijo/a caracterizadas por la imitación del balbuceo, la elicitación de vocalizaciones, la propuesta de significados, los turnos de palabras, la aparición de protoconversaciones, etc. Sin embargo, actualmente muchos bebés pasan mucho tiempo con otros cuidadores distintos a la madre como, por ejemplo, padres, tíos/as, abuelos/as y educadores/as de centros educativos, encontrándose también efectos positivos de los distintos cuidadores sobre el desarrollo del lenguaje infantil (Farkas, 2011; Hoff, 2006; Pancsofar \& Vernon-Feagans, 2006; 2010). Recientemente, por ejemplo, están apareciendo investigaciones como las de Pancsofar \& 
Vernon-Feagans $(2006 ; 2010)$ dónde se evidencia que aunque los padres y las madres se comprometen e interactúan de manera diferente con sus niños/as, los padres juegan un papel importante en el desarrollo comunicativo de los mismos.

El presente trabajo tiene por objetivo aplicar a bebés de entre 8 y 15 meses el apartado de "Vocalizaciones" del CDI MacArthur-Bates desarrollado para nuestra lengua por López-Ornat et al. (2005). Nuestros objetivos son múltiples. En primer lugar, pretendemos conocer las producciones vocales tempranas anteriores a la primera palabra a fin de confirmar los resultados ya existentes sobre el patrón evolutivo del desarrollo del lenguaje. En segundo lugar, trataremos de comprobar la fiabilidad del apartado de "Vocalizaciones" mediante el coeficiente alfa de Cronbach para confirmar si este recurso es fiable y válido respecto al desarrollo lingüístico y comunicativo dados los escasos datos existentes al respecto. En tercer lugar, se estudiará qué influencia ejercen determinados factores extralingüísticos como por ejemplo, el sexo, el orden de nacimiento y el nivel educativo materno, en el desarrollo de las vocalizaciones tempranas, esperando volver a confirmar muchos de los resultados encontrados por algunos de los trabajos citados anteriormente. Concretamente, se espera que las diferencias en el desarrollo prelingüístico en edades tan tempranas, entre los niños y las niñas, sean inexistentes. Por el contrario, las vocalizaciones prelingüísticas estarán influenciadas por el nivel de estudios académicos de la madre esperando encontrar un mayor número de vocalizaciones en los niños/as cuyas madres poseen una mayor formación académica. La variable orden de nacimiento también influirá en las vocalizaciones tempranas, siendo estas más elevadas en los hijos/as primogénitos. Por último, se pretende analizar una variable no contemplada por estudios similares previos, en concreto la influencia que ejerce la persona que más está con el niño/a la mayor parte del día (madre vs. otras personas), esperando hallar diferencias significativas en la figura de la madre por ser ésta generalmente la cuidadora principal.

\section{Método}

\section{Participantes}

En el estudio participaron 39 niños (20 niñas y 19 niños) de edades comprendidas entre los 8 y los 15 meses de edad (edad media 12.23 meses; desviación típica 2.58). En la Figura 1 puede verse la distribución de los participantes según su edad y sexo. 


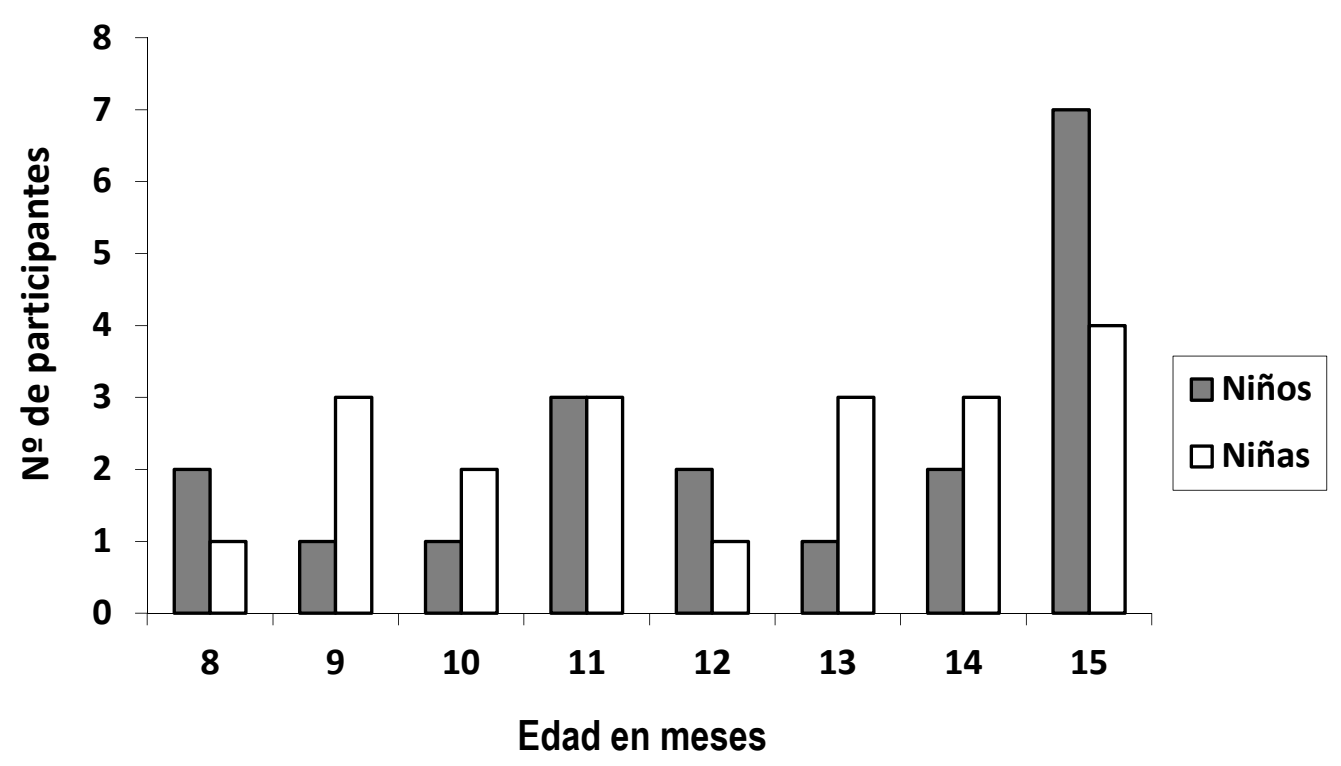

Figura 1. Distribución de los participantes según edad y sexo

Todos los niños eran nacidos a término y presentaban un desarrollo normal sin manifestar problemas de audición o lenguaje (solo un 15,4\% del total de la muestra había tenido alguna vez infecciones de oído). La mayoría de los niños y niñas eran monolingües de español $(84,6 \%)$ y el $15,4 \%$ de los niños y niñas tenían contacto con otras lenguas (ruso, rumano e inglés) desde el momento de su nacimiento. El 84,6\% de las familias procedían del territorio español (Madrid, Salamanca y Ciudad Real) frente a un 10,3\% que venían de otros países de habla no hispana. Con respecto a la persona con la que pasan la mayor parte del tiempo, el 79,5\% lo pasan con la figura materna y el 20,5\% están con personas distintas a la madre (padre, abuelos, tías, etc.). El cuestionario fue rellenado mayoritariamente por las madres $(79,5 \%)$ y en un $20,5 \%$ por los padres. El nivel educativo de las madres en general es medio alto: el 71,9\% tienen estudios universitarios y bachillerato, el $17,9 \%$ tienen al menos estudios de secundaria y el resto $(5,2 \%)$ han cursado solo primaria o no tienen escolarización. Por el contrario, el nivel educativo de los padres es medio bajo: el 51,3\% tienen estudios superiores y bachillerato, el 15,4\% cursó estudios de secundaria y el resto tienen estudios de primaria o no han recibido escolarización. Finalmente, el 48,7\% de los participantes han nacido en primer lugar, el $30,8 \%$ han nacido en segundo lugar y el 17,9\% en tercer lugar. El 53,8\% de las familias tienen más de un hijo/a y el 46,2 \% solo un hijo/a. 
Los participantes del presente estudio fueron reclutados de diferentes centros de Educación Infantil tanto públicos como privados de la Comunidad Autónoma de CastillaLa Mancha, concretamente de la provincia de Toledo.

\section{Instrumentos}

Como se ha indicado, López-Ornat et al., (2005) llevaron a cabo la adaptación del Inventario de Desarrollo Comunicativo MacArthur-Bates para el español de España. El Inventario consta de dos formas, la forma I, denominada "Vocalizaciones, primeras palabras y gestos" y la forma II, denominada "Vocalizaciones, palabras y gramática" que se aplican a niños/as de 8 a 15 y de 16 a 30 meses, respectivamente. La estructura del Inventario de Desarrollo Comunicativo MacArthur-Bates en su versión para el español de España es básicamente la misma que la versión original si bien presenta algunas novedades y variaciones. Un cambio importante en la versión española es la incorporación de un apartado nuevo denominado "Vocalizaciones" que viene a completar y enriquecer la evaluación de las vocalizaciones prelingüísticas. Se trata en definitiva de enriquecer la evaluación temprana de las habilidades comunicativas-lingüísticas en los niños/as de entre 8 y 15 meses y más allá de esta edad dado que la forma II también incluye el apartado "Vocalizaciones".

Este nuevo apartado "Vocalizaciones" incluye 12 ítems (ver Tabla 1) referidos a conductas vocales precedentes a la emergencia de las primeras palabras y fácilmente reconocibles por los padres y madres (López-Ornat et al., 2005; Mariscal et al., 2007). En este apartado, se pregunta a los padres si su hijo/a de 8-15 meses hace ese tipo de conductas prelingüísticas. Se ofrecen a los padres y madres 3 posibles respuestas a cada pregunta: "todavía no" para vocalizaciones cuya producción nunca han observado; " $a$ veces" para las que su hijo/a está produciendo actualmente pero con frecuencia reducida o escasa y "muchas veces" para conductas que se repiten frecuentemente.

Al final del Inventario aparece una sección de información general (López-Ornat eta al., 2005) que también se ha utilizado en el presente trabajo. Esta sección recoge datos tanto del niño/a como de los padres. Los datos del niño/a se refieren a la edad, sexo, orden de nacimiento, persona con la que pasa la mayor parte del tiempo, escolarización, contacto con otras lenguas y cuestiones relativas al estado de salud. Con respecto a los datos de los padres, se recoge información acerca de quién rellena el cuestionario, escolaridad de cada uno y lugar de origen. 
Tabla 1. Descripción de los ítems del apartado "Vocalizaciones” del CDI MacArthurBates para el español de España.

\begin{tabular}{ll}
\multicolumn{1}{c}{ Ítem } & \multicolumn{2}{c}{ Descripción } \\
\hline $\mathbf{1}$ & Atención, interés y trabajo de estímulos auditivos y musicales \\
$\mathbf{2}$ y 3 & Vocalizaciones que acompañan las primeras manifestaciones \\
& referenciales protoimperativas y protodeclarativas \\
$\mathbf{4}$ & Protoconversaciones \\
$\mathbf{5}$ y 6 & Desarrollo segmental (balbuceo canónico y variado) \\
$\mathbf{7}$ y 8 & Imitación de palabra y entonación \\
$\mathbf{9}$ y $\mathbf{1 0}$ & Desarrollo vocalizador en contextos no comunicativos \\
$\mathbf{1 1}$ & Desarrollo suprasegmental (jerga); protonarrativa \\
$\mathbf{1 2}$ & Protopalabras
\end{tabular}

\section{Procedimiento}

Se concertó una reunión con el director/a en cada uno de los centros de Educación Infantil que participaron en el estudio para explicar cuál era su objetivo y el procedimiento que se llevaría a cabo en caso de acceder a la colaboración. Posteriormente, se redactó una carta dirigida a los padres y madres con bebés en edades comprendidas entre los 8 y los 15 meses de edad, explicando el objetivo del estudio y pidiendo la colaboración de los mismos. Todos los padres debían firmar un consentimiento informado en el que se garantizaba la protección y reserva de la información obtenida con fines de investigación. Por último, para facilitar tanto el anonimato de los y las participantes como para evitar la pérdida de cuestionarios, se entregó un sobre al director/a de los centros de Educación Infantil que contenía el apartado "Vocalizaciones" del CDI MacArthur-Bates español (López-Ornat et al., 2005) junto con la sección de información general para que se entregara a los padres y madres participantes en el estudio. Transcurridos 15 días, se recogieron los sobres rellenos, con los inventarios cumplimentados. No fue necesario explicar a los padres y madres del estudio cómo rellenar el inventario porque en éste aparecen unas instrucciones muy claras de cómo debe hacerse.

Una vez cumplimentados los inventarios se procedió a la corrección y puntuación de los mismos para la interpretación y análisis de los resultados. Siguiendo a Lopez-Ornat et al. (2005), se convirtieron las repuestas de los cuestionarios en puntuaciones, de manera que la respuesta "todavía no" se puntuaba como 0 , mientras que la repuesta "a veces" o 
“muchas veces" se puntuaban como 1 . Se contaban todas las puntuaciones siendo la puntuación mínima 0 y la máxima 12.

\section{Resultados}

A continuación se presentan los resultados obtenidos tras la realización de los análisis estadísticos mediante el programa estadístico SPSS versión 19.00. En primer lugar, se comprobó si las variables a analizar estadísticamente seguían una distribución de normalidad mediante la prueba K-S de normalidad. Solamente las variables edad de los participantes y puntuación global en el apartado "Vocalizaciones" siguen la distribución normal. Por este motivo, se utilizaron pruebas no paramétricas para los pertinentes análisis estadísticos. Se ha tenido en cuenta para todos los análisis estadísticos el nivel de confianza del .05.

El apartado "Vocalizaciones" presenta una elevada consistencia interna, a la vista de las puntuaciones obtenidas con la prueba $\alpha$ de Cronbach. El valor obtenido fue de .82 . El cálculo del coeficiente de fiabilidad $\alpha$ de Cronbach ítem a ítem, también revela un elevado grado de consistencia interna. Concretamente la descripción por ítems fue la siguiente: ítem 1) .82, ítem 2) .82, ítem 3) .81, ítem 4) .81, ítem 5) .81, ítem 6) .81, ítem 7) .8, ítem 8) .81, ítem 9) .8, ítem 10) .81, ítem 11).81e ítem 12) .81.

En relación con la edad, el análisis de las puntuaciones en el apartado "Vocalizaciones" ha mostrado una correlación positiva (correlación de Pearson) entre esta y la puntuación total obtenida en dicho apartado $(\mathrm{r}=.39, p \leq .01)$. Por ítems, el coeficiente de la correlación de Sperman, muestra como la edad también ha tenido una correlación significativa en los ítems $2(\mathrm{r}=.57, p \leq .001), 7(\mathrm{r}=.39, p \leq .012)$ y $12(\mathrm{r}=.65$, $p \leq .001)$.

La Figura 2 representa gráficamente el patrón evolutivo en el desarrollo de las producciones vocálicas teniendo en cuenta las puntuaciones medias en el apartado de "Vocalizaciones" en cada nivel de edad. Puede observarse cómo la puntuación media a los 8 meses se sitúa por encima de 6 puntos (de un máximo de 12). Sin embargo, a la edad de 11-12 meses y a los 14 meses se produce un descenso en esta evolución. 


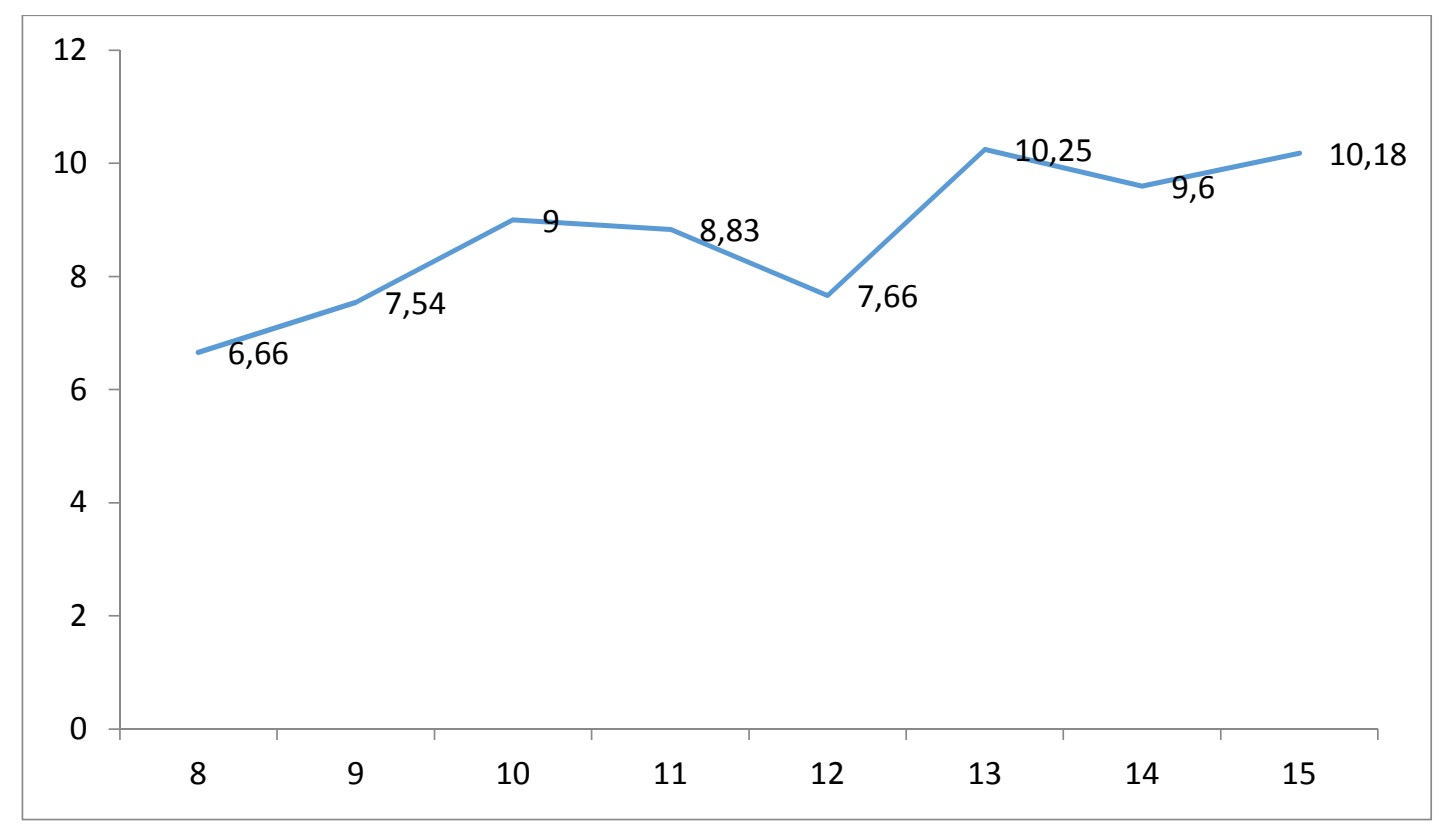

Figura 2. Puntuaciones medias en el apartado "Vocalizaciones" (eje de abcisas) por edad -eje de ordenadas

En cuanto a la influencia de la variable sexo, en los análisis estadísticos efectuados solo se ha hallado una diferencia significativa entre niños y niñas (prueba no paramétrica U de Mann-Whitney) sobre el ítem 8 "imitación de entonación” ( $\mathrm{Z}=-2.36, p \leq .018)$ siendo mayor el rango promedio de niñas que de niños (rango promedio de 23.63 frente a 16.18).

Otra de las variables objeto de este trabajo se refiere al orden de nacimiento. Por lo que respecta a esta variable, los análisis estadísticos realizados (prueba U de MannWhitney) muestran solamente una relación significativa sobre el ítem 3 "protodeclarativo" $(\mathrm{Z}=-2.768, p \leq .006)$, siendo superior el rango promedio entre los primogénitos frente a los sujetos que tienen hermanos/as (rango promedio de 23.24 frente al 15.76).

Con respecto a la influencia de la variable con quién pasa el niño/a la mayor parte del día (madre vs. otras personas), los análisis estadísticos llevados a cabo (prueba no paramétrica U de Mann-Whitney), reflejan un resultado relativamente sorprendente e inesperado. Ha aparecido una relación significativa sobre la puntuación total obtenida en el apartado "Vocalizaciones" $(Z=-2.094, p \leq .036)$ siendo el rango promedio mayor en las personas distintas a la madre (27.44 frente al 18.08). En este estudio, la madre es la cuidadora principal del niño/a al ser la persona que pasa más tiempo con el niño/a durante el día (79,5\%). 
Finalmente, se comprobó el efecto dela variable estatus socioeconómico familiar, tomándose como indicador el nivel educativo de la madre (alta formación académica vs. baja). Los análisis estadísticos efectuados con la prueba no paramétrica U de MannWhitney muestran diferencias estadísticamente significativas en el ítem 1 "atención, interés y trabajo de estímulos auditivos y musicales" ( $\mathrm{Z}=-2.24, p \leq .025), 4$ "protoconversación" $(\mathrm{Z}=-2.151, p \leq .032)$ y 11 "jerga" $(\mathrm{Z}=-2.278, p \leq .023)$. En los ítems 1 y 4 la diferencia es a favor de las madres con alta formación académica (21.11 frente al 12.44 para el ítem 1 y 20.88 frente 13.17 para el ítem 4). En el ítem 11, la diferencia es a favor de las madres con menor nivel educativo (25.67 frente al 16.86).

\section{Discusión}

Los datos obtenidos con la aplicación de la prueba $\alpha$ de Cronbach indican que el apartado "Vocalizaciones" presenta unos buenos índices de consistencia interna tanto a nivel general como ítem por ítem, alcanzando globalmente una puntuación de .82. Este dato es superior al encontrado por el equipo de López-Ornat et al. (2005) que aun siendo elevado, fue de .70. Dada la escasez de datos a este respecto, puede afirmase que el apartado "Vocalizaciones" muestra una elevada fiabilidad en la evaluación del desarrollo prelingüístico infantil.

Distintos autores avalan la existencia de un patrón evolutivo en el que se observa un mayor desarrollo de las vocalizaciones según avanza la edad de los bebés. Además, este desarrollo es temprano, lento, gradual y continuo (Karousou, 2003; López-Ornat et al., 2005; Mariscal et al., 2007). Nuestros datos también muestran una relación entre la edad y la puntuación directa en el apartado de "Vocalizaciones", confirmándose la existencia de este paulatino desarrollo lineal, progresivo y temprano. Sin embargo, observamos un descenso en esta evolución a los 11-12 meses y a los 14 meses. Este dato puede explicarse por el tamaño tan pequeño de la muestra que la hace poco representativa. En este sentido, tal y como sugieren Ambrose et al. (2014), Guzzetta (2014), Karousou and López-Ornat (2013) y López-Ornat (2011) no se debe desdeñar la importancia de las diferencias individuales que caracteriza estos aprendizajes lingüísticos tempranos.

Por lo que respecta a las distintas conductas vocálicas examinadas, nuestros resultados ponen de manifiesto que según aumenta la edad, los niños/as presentan mayores emisiones protoimperativas. Este dato confirmaría los encontrados en otros trabajos acerca de la emergencia del gesto de señalar para pedir las cosas como precursor del lenguaje (Delgado, Gómez y Sarriá, 2010; Esteve-Gibert \& Prieto, 2014; Kirk, 
Howlett, Pine, \& Fletcher, 2013; Murillo \& Belinchón, 2013). También con la edad, los sujetos de nuestra muestra producen más imitación de palabras y protopalabras, vocalizaciones todas ellas indicadoras de una progresiva especialización comunicativalingüística más cercana a la emisión de la primera palabra (López-Ornat \& Karousou, 2005; Stoel-Gammon, 2011).

Con respecto a las variables que inciden en el desarrollo prelingüístico y comunicativo temprano, una de las contempladas en este trabajo es el sexo. Como se indicó en la introducción, existe una controversia respecto a su influencia. Nuestros resultados, como habíamos predicho, apoyan los resultados de Jackson-Maldonado et al. (2013), López-Ornat et al. (2005) y Mariscal et al. (2007) de modo que no hemos encontrado diferencias significativas en el desarrollo de las vocalizaciones tempranas entre niños y niñas. De este modo, parece que en los primeros estadios del desarrollo lingüístico no existen diferencias de sexo, sobre todo cuando el desarrollo del lenguaje es incipiente. Pese a ello, sí se ha hallado una diferencia significativa en las niñas en el ítem 8 “imitación de entonación”. Este ítem hace referencia a la repetición inmediatamente después de oír una frase, como por ejemplo una pregunta, de su entonación y su melodía. Los aspectos prosódicos, de entonación, de ritmo, de melodía, de acentuación y de segmentación han sido estudiados por algunos investigadores (Crespo, Kaland, Swerts, \& Prieto, 2013; Esteve-Gibert \& Prieto, 2014; Falcón, Alva \& Franco, 2013; Guzzetta, 2014; Karousou \& López-Ornat, 2013; Prieto, Borrás-Comes, Tubau, \& Espinal 2013) considerándose aspectos importantes y necesarios antes de la producción de las primeras palabras propiamente dichas. La mayor superioridad de las niñas en estos aspectos, puesta de manifiesto por nuestros resultados, sugeriría que algunas de las habilidades prosódicas necesarias para el desarrollo lingüístico posterior se desarrollan antes en las niñas, si bien, este resultado ha de tomarse con cautela dado el tamaño de la muestra.

En relación con la variable orden de nacimiento, en línea con algunos datos previos en la literatura (Benigno et al., 2007; Galián et al., 2010; Hoff, 2006; Malmeer \& Assadi, 2013; Pancsofar \& Vernon-Feagans, 2010; Pérez \& García, 2003; Pérez-Pereira \& Resches, 2007; Schults et al., 2012), observamos una pequeña ventaja lingüística en los niños/as nacidos en primer lugar frente aquellos que tienen hermanos/as mayores. No obstante, esta ventaja puede considerarse de baja magnitud ya que únicamente hemos encontrado una diferencia significativa en los primogénitos en el ítem 3 "protodeclarativo". Este ítem se refiere a cómo los niños/as a veces acompañan sus gestos con sonidos para llamar la atención sobre algo que les atrae. La aparición de este signo 
prelingüístico podría constituir, sino una condición para el desarrollo de las primeras palabras, sí al menos un importante facilitador como sugieren diferentes autores (Delgado et al., 2010; Esteve-Gibert \& Prieto, 2014; Kirk et al., 2013; Murillo \& Belinchón, 2013). De este modo, los hijos/as primogénitos no solo llamarían la atención sobre algo que les atrae acompañando sus gestos con sonidos, sino que suponemos que recibirían mayor atención por parte de sus progenitores al poder dedicarles más tiempo como proponen algunos autores (Benigno et al., 2007; Galián et al., 2010; Hoff, 2006), si bien, este aspecto habrá que estudiarlo en el futuro.

Los resultados obtenidos en la variable con quién pasa la mayor parte del tiempo el niño/a (con la madre vs. con otras personas como padres, abuelos/as, etc.), son sorprendentes al haber podido comprobar que los cuidadores distintos a la madre y con los que pasan menos tiempo son los que informan de un mayor número de vocalizaciones en los niños/as. A pesar de la elevada evidencia que existe acerca de la alta fiabilidad y validez de los padres y madres como informantes del desarrollo lingüístico de sus hijos/asen la infancia temprana (Bates et al., 1979; Guiberson et al., 2011; Karousou \& López-Ornat, 2013; Marcham \& Martinez-Sussmann, 2002; Mariscal et al., 2006; Mariscal et al., 2010; Thal et al., 2000), tras nuestro resultado podemos decir que los padres u otros cuidadores de los menores reconocen e informan de manera diferente a como lo hacen las madres. Como sugiere Farkas (2011), los padres pueden tender a sobredimensionar las vocalizaciones en sus hijos/as de manera que sus observaciones podrían presentar un sesgo positivo, tanto cognitivo como afectivo. Ello se debería a que el conocimiento que tienen de su hijo/a es diferente al observar a sus hijos/as en situaciones cotidianas distintas a las de la madre. De confirmarse este resultado, un aspecto a tener en consideración por investigaciones futuras debería ser averiguar la influencia de la variable del cuidador principal (madre vs. otras personas significativas tales como padres, abuelos/as, educadores/as) para conocer el papel diferenciador que ejercen en la observación del desarrollo prelingüístico infantil con el fin de aumentar la objetividad de los comportamientos lingüísticos observados.

En relación con el nivel educativo de las madres, tal como se predijo, los hijos/as de las madres con mayor nivel educativo muestran puntuaciones más altas únicamente en determinadas conductas vocálicas del apartado "Vocalizaciones". Más concretamente, estas puntuaciones superiores se dieron en los ítems "atención, interés y trabajo de estímulos auditivos y musicales" y “protoconversación”. El primer ítem se refiere a si los niños/as cantan por su cuenta tras oír cantar a un adulto o a algún muñeco. Este resultado 
parece indicar que las madres que tienen una elevada formación académica pondrían en marcha con sus bebés intercambios comunicativos caracterizados por la estimulación sonora a través del canto, las canciones, las canciones de cuna, los juegos musicales, etc., ejemplos muy relacionados con la música, el ritmo, la melodía, la segmentación y la prosodia. Este resultado, apoyaría la musicalidad comunicativa (Malloch, 2000) como herramienta importante y necesaria antes de la producción de las primeras palabras (Esteve-Gibert \& Prieto, 2014; Guzzetta, 2014; Karousou \& López-Ornat, 2013; Malloch, 1999/2000; Prieto et al., 2013) de manera que estos bebés tendrían una progresiva acumulación de conocimientos articulatorios prosódicos que podríamos considerar prerrequisitos necesarios para el desarrollo lingüístico posterior (Lopéz-Ornat et al., 2005).

El ítem "protoconversación” también es significativo estadísticamente a favor de las madres de nivel formativo elevado. Este ítem se produce cuando al hablar a los niños/as estos parece que responden y establecen un diálogo o conversación, no mediante palabras, sino con sonidos, grititos, sonidos parecidos a canciones, etc. Este dato podría explicarse por el hecho de que las madres de nivel educativo alto utilizan el lenguaje para hablar más a sus hijos/as, provocar la conversación y el diálogo, comunicar intenciones entre madre e hijo/a y llevar a cabo intercambio de turnos (Hoff, 2006).

Por otro lado, nuestro trabajo ha puesto de manifiesto que las madres de nivel educativo inferior obtienen puntuaciones mayores en el ítem "jerga". Este ítem se produce cuando los niños/as hablan sin usar palabras pareciendo que han hecho una pregunta o que han regañado a alguien o que se han asombrado de algo e incluso a veces puede incluir alguna palabra reconocible. La jerga tiene una apariencia de conversación que será reemplazada posteriormente por la expresión verbal de la primera palabra propiamente dicha. Nuestros resultados reflejarían que tanto en los niveles educativos maternos superiores como en los inferiores, aparecen determinadas conductas prevocálicas articulatorias caracterizadas por aspectos prosódicos y melódicos (canto, protoconversación y jerga) muy necesarias para, posteriormente, la emergencia de la palabra y la capacidad simbólica. No obstante, como argumenta Ramsdell et al., (2012), la intensidad y la frecuencia de las vocalizaciones difiere en función del nivel educativo y/o cultural de las familias llegando a ser diferencias más cuantitativas que cualitativas.

En síntesis, a la luz de estos resultados podemos concluir que en el presente trabajo se refleja la compleja interacción e influencia de múltiples factores extralingüísticos en el desarrollo de las vocalizaciones en los niños/as de 8-15 meses de edad. Se confirman los 
hallazgos realizados en estudios previos, sobre el patrón evolutivo del desarrollo del lenguaje. Igualmente, se refuerza la utilidad y fiabilidad del apartado "Vocalizaciones" en la evaluación del desarrollo prelingüístico.

Por último, el presente trabajo cuenta con algunas limitaciones que hay que tener en cuenta para la interpretación de los resultados. En primer lugar, el tamaño de la muestra puede considerarse reducida y poco representativa teniendo en cuenta la enorme variabilidad individual inherente al propio desarrollo lingüístico en sus primeros estadios. Por ello, los resultados han de interpretarse con precaución. En segundo lugar, sólo se ha evaluado el apartado "Vocalizaciones" con la consiguiente limitación en los datos obtenidos y analizados que se ciñen exclusivamente a dicho apartado y período (8-15 meses). Sería conveniente ampliar el periodo de edad, así como, el resto de apartados del cuestionario para poder conocer la influencia de los fenómenos analizados en el desarrollo comunicativo-lingüístico posterior. En tercer lugar, la percepción de las conductas prelingüísticas observadas por los cuidadores significativos del niño/a es un proceso muy complejo que puede estar sujeto a sesgos observacionales. Por ello, se recomienda utilizar más de un procedimiento de evaluación, así como, diferentes informantes del desempeño lingüístico (Farkas, 2011). En particular, parece necesario seguir investigando los diferentes estilos de informar sobre el desarrollo lingüístico y comunicativo de las distintas personas que tengan relación con los bebés y no solo del padre o de la madre. Esto es más importante en la sociedad actual en la que son diversas las otras personas que en determinados momentos ejercen el papel de cuidador significativo (abuelos/as, educadores/as, etc.).

\section{Bibliografía}

Ambrose, S., Unflat, L., Walker, E., Harrison, M., Oleson, J., \& Moeller, M (2014). Speechsound production in 2-year-olds who are hard of hearing. American Journal of Speech-Language Pathology, 23, 91-104.

Bates, E., Benigni, L., Bretherton, I., Camaioni, L., \& Volterra, V. (1979). The emergence of symbols: Cognition and communication in infancy. Cambridge University Press.

Benigno, J., Clark, L., \& Farrar, J. (2007). There is not always a crowd: contexts of joint attention and language. Journal of Child Language, 34, 175-187. 
Bouchard, C., Trudeau, N., Sutton, A., Boudreault, M.C., \& Deneault, J. (2009). Gender differences in language development in French Canadian children between 8 and 30 months of age. Applied Psycholinguistics, 30, 685-707.

Bruce, B., Kornfalt, R., Radeborg, K., Hansson, K., \& Nettelbladt, U. (2003). Identifying children at risk for language impairment: screening of communication at 18 months. Acta Paediatrica, 92, 1090-1095.

Crespo, V., Kaland, C., Swerts, M., \& Prieto, P. (2013). Perceiving incredulity: The role of intonation and facial gestures. Journal of Pragmatics, 47, 1-13.

Delgado, B., Gómez, J.C., y Sarriá, E. (2010). Funciones tempranas del gesto de señalar privado: La contemplación y la autorregulación a través del gesto de señalar. Acción Psicológica, 7, 59-70.

DePaolis, R., Vihman, M.M., \& Nakai, S. (2013). The influence of babbling patterns on the processing of speech. Infant Behavior \& Development 36, 642-649.

D’Odorico, L., Majorano, M., Fasolo, M., Salerni, N., \& Suttura, C. (2011). Characteristics of phonological development as a risk factor for language development in Italian-speaking pre-term children: A longitudinal study. Clinical Linguistics \& Phonetics, 25, 53-65.

Esteve-Gibert, N., \& Prieto, P. (2014). Infants temporally coordinate gesture-speech combinations before they produce their first words. Speech Communication 57, 301-316.

Falcón, A., Alva, E., y Franco, A. (2013). Segmentación y categorización intraléxica por infantes aprendices del español de 9 a 12 meses de edad. Psicológica, 34, 37-58.

Farkas, C. (2011). Inventario del Desarrollo de Habilidades Comunicativas McArthurBates (CDI): propuesta de una versión abreviada. Universitas Psychologica, 10, 245-262.

Fenson, L., Dale, P.S., Reznick, J.S., Thal, D., Bates, E., Harthug, J.et al., (1993). The MacArthur Communicative Development Inventories: User's guide and technical manual. San Diego, Singular Publishing Group.

Furey, J. (2011). Production and maternal report of 16 and 18 month olds' vocabulary in low and middle income families. American Journal of Speech Language Pathology, 20, 38-46.

Galeote, M., Soto, P., Serrano, A., Pulido, L., Rey, R., \& Martínez-Roa, P. (2006). Un nuevo instrumento para evaluar el desarrollo comunicativo y lingüístico de niños con síndrome de Down. Revista Síndrome de Down, 23, 20-26. 
Galeote, M., Soto, P., Sebastián, E., Rey, R., \& Checa, E. (2012). La adquisición del vocabulario en niños con síndrome de Down: datos normativos y tendencias de desarrollo. Infancia y Aprendizaje, 35, 111-122.

Galián, M.D., Ato, S., \& Carranza, J.A. (2010). Explosión del vocabulario infantil y primer lenguaje: una revisión. Anales de Psicología, 26, 2, 341-347.

García, I., Arratibel, N., Barreña, A., \& Exeizabarrena, M.J. (2008). Adaptación de los inventarios MacArthur-Bates al euskera: desarrollo comunicativo entre los 8-30 meses. Infancia y Aprendizaje, 31, 411-424.

Guiberson, M., \& Rodríguez, B. (2010). Measurement properties and classification accuracy of two Spanish parent surveys of language development for preschoolage children. American Journal of Speech-Language Pathology, 19, 225-237.

Guiberson, M., Rodríguez, B., \& Dale, P. (2011). Classification accuracy of brief parent report measures of language development in Spanish-speaking toddlers. Language, Speech and Hearing Services in Schools, 42, 536-549.

Guzzetta, F. (2014). Behavioral assessment of language brain processing in the first year of life. European Journal of Paediatric Neurology, 18, 551-557.

Hoff, E. (2006). How social contexts support and shape language development. Development Review, 26, 55-88.

Hurtado, N., Marchman, V., \& Fernald, A. (2008). Does input influence up take? Links between maternal talk, processing speed and vocabulary size in Spanish-learning children. Developmental Science 11, 31-39.

Jackson-Maldonado, D., Thal, D., Fenson, L., Marchman, V.A., Newton, T., \& Conboy, B. (2003). MacArthur Inventarios del desarrollo de habilidades comunicativas. User's Guide and Technical Manual. Baltimore, P.H. Brookes.

Jackson-Maldonado, D., Marchman, V., \& Fernald, L. (2013). Short-form versions of the Spanish MacArthur-Bates Communicative Development Inventories. Applied Psycholinguistics 34, 837-868.

Karousou, A. (2003). Análisis de las vocalizaciones tempranas: su patrón evolutivo y su función determinante en la emergencia de la palabra. Tesis doctoral. Servicio de Publicaciones de la Universidad Complutense de Madrid. Facultad de Psicología.

Karousou, A., \& López-Ornat, S. (2013). Prespeech vocalitaions and the emergence of speech: a study of 1005 Spanish children. Spanish Journal of Psychology,16, 132. 
Kirk, E., Howlett, N., Pine, K., \& Fletcher, B. (2013). To sign or not to sign? The impact of encouraging infants to gesture on infant language and maternal mindmindedness. Child Development, 84, 574-590.

Lara, M.F., Gómez, A.M., Gálvez, D.M., Mesa, C., \& Serrat, E. (2011). Normativización del Inventario del Desarrollo Comunicativo MacArthur-Bates al español, Colombia. Revista Latinoamericana de Psicología, 43, 241-254.

López-Ornat, S. (2011). La adquisición del lenguaje, un resumen en 2011. Revista de investigación en logopedia, 1, 1-11.

López-Ornat, S., Gallego, C., Gallo, P., Karousou, A., Mariscal, S., \& Martínez, M. (2005). Inventario de Desarrollo Comunicativo MacArthur. Manual. Madrid: TEA Ediciones.

López-Ornat, S., \& Karousou, A. (2005): Las vocalizaciones tempranas (8-30 meses) y su relación con el vocabulario y la gramática. Su medida en el "CDI español": resultados preliminares. En Ma . A. Mayor, B. Zubiauz \& E. Díez-Villoria (Eds.), Estudios sobre la adquisición del lenguaje (pp. 401-420) Salamanca: Universidad de Salamanca.

Malloch, S. (2000). Mothers and infants and communicative musicality. Musicae Scientiae, 3, 29-57.

Malmeer, E., \& Assadi, N. (2013). Language production and comprehension: The effect of Pre-school aged siblings on toddlers language development. Theory and Practice in Language Studies, 3, 7, 1226-1231.

Marcham, V.A., \& Martinez-Sussmann, C. (2002). Concurrent validity of caregiver/parent report measures of language for children who are learning both English and Spanish. Journal of Speech, Language and Hearing Research, 45, 983-997.

Mariscal, S., \& Gallo, P. (2006). La evaluación del desarrollo gramatical temprano. Estudios de Psicología, 27, 153-173.

Mariscal, S., Karousou, A., Martínez, M., Gallo, P., Gallego, C., \& López-Ornat, S. (2007). ¿Es posible evaluar el desarrollo del lenguaje de los bebés? Infocop Online, 6 julio: ISSN: 1886-1385

Mariscal, S., López-Ornat, S., Gallego, C., Gallo, P., Karousou, A., \& Martínez, M. (2007). La evaluación del desarrollo comunicativo y lingüístico mediante la versión española de los inventarios MacArthur-Bates. Psicothema, 19, 190-197. 
Mariscal, S., Nieva, S., Gallego, C., \& López-Ornat, S. (2006). La fiabilidad de los padres como informantes del conocimiento gramatical de sus hijos: Los inventarios de MacArthur en Español. En B. Gallardo, C. Hernández, \& V. Moreno (Eds.), Lingüística clínica y neuropsicología cognitiva. Actas del I Congreso Nacional de Lingüística Clínica (pp. 170-181).Valencia.

Mariscal, S., Nieva, S., \& López-Ornat, S. (2010). Observar y medir el desarrollo gramatical temprano en español. Psicothema, 22, 51-56.

Murillo, E., y Belinchón, M. (2013). Patrones comunicativos multimodales en la transición a las primeras palabras: cambios en la coordinación de gestos y vocalizaciones. Infancia y Aprendizaje, 36, 473-487.

Oller, D. K. (2000). The Emergence of the Speech Capacity. Mahwah, NJ, US: Lawrence. Erlbaum Associates, Inc., Publishers.

Owens, R. (2003). Desarrollo del lenguaje. Madrid. Pearson. Prentice hall.

Pancsofar, N., \& Vernon-Feagans, L. (2006). Mother and father language input to young children: Contributions to later language development. Journal of Applied Developmental Psychology, 27, 571-587.

Pancsofar, N., \& Vernon-Feagans, L. (2010). Father's early contributions to children's language development in families from low-income rural communities. Early Childhood Research Quarterly, 25, 450-463.

Pérez, M., \& García, P. (2003). El diagnóstico del desarrollo comunicativo en la primera infancia: adaptación de las escalas MacArthur al gallego. Psicothema, 15, 352361.

Pérez-Pereira, M., \& Resches, M. (2007). Elaboración de las formas breves del Inventario do Desenvolvemento de Habilidades Comunicativas. Datos normativos y propiedades psicométricas. Infancia y Aprendizaje, 30, 565-588.

Prieto, P., Borrás-Comes, J., Tubau, S., \& Espinal, M.T. (2013). Prosody and gesture constrain the interpretation of double negation. Lingua, 131, 136-150.

Ramsdell, H., Oller, D. K., Buder, E., Ethington, C., \& Chorna, L. (2012). Identification of prelinguistic phonological categories. Journal of Speech, Language and Hearing Research, 55, 1626-1639. doi: 10.1044/1092-4388(2012-11-0205).

Schults, A., Tulviste, T., \& Konstabel, K. (2012). Early vocabulary and gestures in Estonian children. Journal of Child Language, 39, 664- 686.

Serra, M., Serrat, E., Solé, R., Bel, A., \& Aparici, M. (2013). La adquisición del lenguaje. Barcelona. Ed.: Ariel. 
Stoel-Gammon, C. (2011). Relationships between lexical and phonological development in young children. Journal of Child Language, 38, 1-34.

Thal, D., Jackson-Maldonado, D., \& Acosta, D. (2000). Validity of parent-report measure of vocabulary and grammar for Spanish-speaking toddlers. Journal of Speech, Language and Hearing Research, 43, 1087-1100. 